\title{
Mortalidade por neoplasias no Brasil (1980/1983/1985): agrupamento dos Estados, comportamento e tendências
}

\author{
Mortality from neoplasms in Brazil (1980/1983/1985): grouping by State, \\ behaviors and tendencies
}

\author{
Fábio Gonçalves Pinto*, Paulo Roberto Curi*
}

\begin{abstract}
PINTO, F.G. \& CURI, P.R. Mortalidade por neoplasias no Brasil (1980/1983/1985): agrupamento por Estados, comportamento e tendencias. Rev. Saúde públ. , S. Paulo, 25:276-81, 1991. Examinou-se a mortalidade por neoplasias no Brasil, utilizando-se dados oficiais do Ministério da Saúde, abrangendo 26 Unidades da Federação e 13 diferentes localizações neoplásicas, para os anos de 1980, 1983 e 1985. As Análises de Agrupamento e de Componentes Principais revelaram comportamento heterogêneo entre regiōes do país, com relação às 13 variáveis estudadas, sendo que os principais elementos discriminantes foram as neoplasias malignas da traquéia/brônquio/pulmão, seguidas das do estômago, esôfago, cólon e pâncreas. Análises complementares evidenciaram tendência de crescimento das taxas de mortalidade para as neoplasias malignas da próstata $(17,74 \%)$, da traquéia/brônquio/pulmão( $15,22 \%)$, da mama $(11,32 \%)$, do pâncreas $(10,23 \%)$, do cólon $(8,08 \%)$, do colo uterino $(6,45 \%)$ e da laringe $(6,36 \%)$. Houve redução da mortalidade por neoplasias benignas/carcinoma "in situ"/ outras $(27,37 \%)$, por neoplasias malignas no reto sigmóide/ânus $(7,67 \%)$, do estômago $(5,31 \%)$, de outro local do útero não especificado $(2,56 \%)$, por leucemia $(0,70 \%)$ e por neoplasias malignas do esôfago $(0,44 \%)$. As neoplasias malignas do estômago foram a principal causa de morte por câncer no Brasil, representando $21,30 \%$ do total médio, seguidas das neoplasias malignas da traquéia/brônquio/pulmão(17,49\% do total médio). Destacam-se os altos índices de mortalidade por neoplasias malignas do esôfago no Estado do Rio Grande do Sul.
\end{abstract}

Descritores: Neoplasias, mortalidade. Distribuição espacial.

\section{Introdução}

As neoplasias malignas estão entre as principais causas de mortalidade mundial, apresentando niveis crescentes nas últimas décadas na maioria dos países (WHO ${ }^{16}, 1988$; Solis e col. $\left.{ }^{15}, 1986\right)$.

Os padrões de ocorrências de neoplasias malignas em diferentes regiōes do mundo apresentam, atualmente, grandes variações com os países em desenvolvimento, em geral, mostrando taxas brutas de incidência mais baixas que aquelas detectadas em nações desenvolvidas (Parkin e col. ${ }^{10}, 1984$ ).

As estatísticas de mortalidade por neoplasias, embora com algumas limitações (Percy e col. ${ }^{11}$, 1981; Lilienfeld e col. $\left.{ }^{5}, 1967\right)$, possibilitam segundo Estevez e col. ${ }^{4}$ (1978), avaliar as tendências no risco de morte por cancêr em relação ao tempo, a possível

\footnotetext{
* Facul dade de Medicina Veterinária e Zootecnia da Universidade Estadual Paulista (UNESP) - Botucatu, SP - Brasil.

Separatas/Reprints:P.R. Curi - Serviço de Estatística e Computação - Campus de Botucatu - Rubião Júnior 18610 - Botucatu, SP - Brasil.
}

Publicação financiada pela FAPESP. Processo Medicina 90/ 4602-1. presença de correlaçð̃es entre o aumento ou a diminuição da mortalidade com aparecimento, aumento ou diminuição de algum fator ambiental concomitante e a comparação das taxas de mortalidade entre diferentes países, regiões ou grupos de populações.

Algumas tendências mundiais têm sido constatadas como, por exemplo, o aumento das taxas de mortalidade por neoplasias malignas em geral (WHO ${ }^{16}$, Possas $^{12}, 1989$ ), a redução da mortalidade por neoplasias malignas do estômago (Montoro e Nogueira ${ }^{9}$ 1983; Campbell ${ }^{2}$, 1980)e o crescimento da participação das neoplasias malignas da mama e da cérvix uterina como causa de morte na população feminina (WHO ${ }^{16}, 1988$ ). Deve ser dado destaque para o incremento nos índices mundiais de mortalidade por neoplasias malignas do aparelho respiratório (Parkin e col. ${ }^{10}, 1984$; Campbell ${ }^{2}, 1980$ ).

Sobre a situação do Brasil nessa área poucas são as informações disponiveis na literatura, destacando-se o trabalho de Brumini ${ }^{1}$ (1982), baseado em dados histopatológicos coletados por laboratórios de diagnóstico anátomo-patológico em um número limitado de municípios. Silva ${ }^{13}$ (1982), por sua vez, apresenta dados obtidos na cidade de Fortaleza - CE, portanto, de interesse prevalentemente regional.

Dados sobre a incidência de neoplasias no Brasil, 
recolhidos pela Organização Mundial da Saúde (Parkin e col. ${ }^{10}, 1984$ ) baseiam-se em levantamentos efetuados em três diferentes áreas: São Paulo, Recife e Fortaleza, não permitindo, portanto, uma avaliação confiável da situação no país como um todo.

Embora reconhecendo que a qualidade da informação possa ser questionada, foram utilizados no presente trabalho os dados oficiais disponiveis para os anos de 1980,1983 e 1985 e para 13 diferentes localizações neoplásicas; propondo-se a examinar os seguintes aspectos:

- a presença de aumento ou de diminuição na mortalidade em cada uma das 26 unidades da Federação e no Brasil como um todo;

- se as tendências observadas são similares nas diferentes regiões do país e em relação a outros países;

- se alguma Unidade da Federação se desvia do padrão geral em relação à qualquer das localizações neoplásicas;

- quais as neoplasias predominantes, qual o peso discriminante de cada uma e a existência ou não de alguma relação entre elas;

- de que forma os Estados se agrupam em função da semelhança em seus perfis de mortalidade para as 13 localizações neoplásicas.

\section{Material e Métodos}

Os dados utilizados foram publicados pelo Ministério da Saúde ${ }^{6,7,8}$ e se referem aos anos de 1980,1983 e 1985 . Os totais absolutos para cada uma das 13 diferentes localizações neoplásicas, em cada uma das 26 Unidades da Federação foram transformados em valores relativos da mortalidade por milhão de habitantes, tomando como base a população dos municípios "com informação regular".

As localizaçōes neoplásicas (variáveis), indicadas pelas abreviaturas que serão utilizadas no presente estudo, estão descritas na seqüência: Esôfago (ESO), Estômago (EST), Cólon (COL), Reto sigmóide/Ânus (RSA), Pâncreas (PAN), Laringe (LAR), Traquéia/Brônquio/Pulmão (TBP), Mama (MM), Colo Uterino (CLU), outro local do Útero não especificado (UNE), Próstata (PRO), Leucemia (LEU), Benignas/Carcinoma "in situ"/ outras neoplasias não especificados (BIVE).

As Unidades (Estados da Federação) utilizadas nas análises, acompanhadas de suas siglas de identificação, foram: Acre (AC), Alagoas (AL), Amapá (AP), Amazonas (AM) , Bahia (BA), Ceará (CE), Distrito Federal (DF), Espírito Santo (ES), Goiás (GO), Maranhão (MA), Mato Grosso (MT), Mato Grosso do Sul (MS), Minas Gerais $(\mathrm{MG})$, Pará (PA), Paraíba (PB), Paraná (PR), Pernambuco (PE), Piauí (PI), Rio Grande do Norte
(RN), Rio Grande do Sul (RS), Rio de Janeiro (RJ), Rondônia (RO), Roraima (RR), Santa Catarina (SC), São Paulo (SP), Sergipe (SE).

Para o estudo estatístico foram utilizados dois métodos multivariados: Análise de Agrupamento e Análise de Componentes Principais (Sneath e So$\mathrm{kal}^{14}, 1973$ ).

A Análise de Agrupamento foi realizada calculando-se a Distância Euclidiana Média entre as Unidades, para o conjunto das 13 variáveis, e utilizando o algorítmo SL(Single Linkage) para a obtenção dos agrupamentos de Estados similares. O resultado da análise foi apresentado em forma gráfica (dendrograma) que auxiliou na identificação dos agrupamentos das Unidades. Foram calculados, ainda, os coeficientes de correlação cofenética entre as distâncias obtidas, considerando-se os pares de anos: $1980 \times 1983,1980 \times 1985$ e $1983 \times 1985$. Quanto maior a concordância entre os agrupamentos de cada par de anos, maior deverá ser o respectivo coeficiente de correlação cofenética calculado.

A Análise de Componentes Principais permite reduzir um conjunto de $\mathrm{p}$ variáveis $(\mathrm{p}=13$, no caso) a um novo conjunto de duas novas variáveis (os componentes principais) de forma tal que cada componente principal seja uma combinação linear das 13 variáveis, capaz de reter grande quantidade da informação fornecida pelo conjunto das variáveis originais. Desta forma, cada uma das Unidades da Federação que era definida por 13 variáveis(13 localizações neoplásicas) passou a ser caracterizada por duas novas variáveis, o que possibilitou sua localização em figuras bidimensionais (ordenação das unidades componentes principais). A adequação desta análise foi verificada pela quantidade da informação total das variáveis originais retida pelos dois componentes principais.

A utilização conjunta dos dendrogramas (da Análise de Agrupamento) e das figuras bidimensionais com a localização dos Estados (Análise de Componentes Principais) permitiu inferir os agrupamentos de unidades similares.

A Análise de Componentes Principais possibilitou, também, verificar a capacidade discriminatória das 13 variáveis originais no processo de agrupamento. Este estudo está relacionado com a contribuição de cada variável para os dois componentes principais, traduzido pelo coeficiente de correlação entre a variável original e o componente principal, que representa o peso da variável no componente. Quanto maior esse peso, maior a capacidade discriminatória da variável.

\section{Resultados e Discussão}

As análises empregadas possibilitaram a divisão 
dos Estados em vários grupos, razoavelmente homogêneos, com os Estados das regiöes Norte, Nordestee parte da Centro-Oeste nitidamente diferenciados dos estados de RS, RJ e SP. As outras Unidades das regiōes Sul e Sudeste, mais a presença ocasional de Estados da região Centro-Oeste, formaram um grupo intermediário. De forma geral, o Estado do Amapá manteve um certo isolamento (exceto em 1980), conforme representadonas Figuras de 1 a 6 .

A composição dos grupos pode ser atribuída de um lado às diferenças regionais no que se refere à eficiência da coleta de informações e na estrutura dos serviços de diagnóstico e de tratamento médicos. Por outro lado, pode significar variações nos índices de mortalidade por neoplasias específicas nos diferentes Estados.

A análise de agrupamento das localizações neoplásicas indicou uma forte homogeneidade de comportamento entre as neoplasias malignas do pâncreas e do colon, com grande concordância nos diferentes anos. O mesmo ocorreu entre as neoplasias malignas de laringe e aquelas do reto/ânus. Estes resultados podem sugerir uma concomitante exposição a fatores predisponentes dessas neoplasias, no conjunto dos Estados da Federação. Em relação ao aparelho digestivo, por exemplo, relação concor-

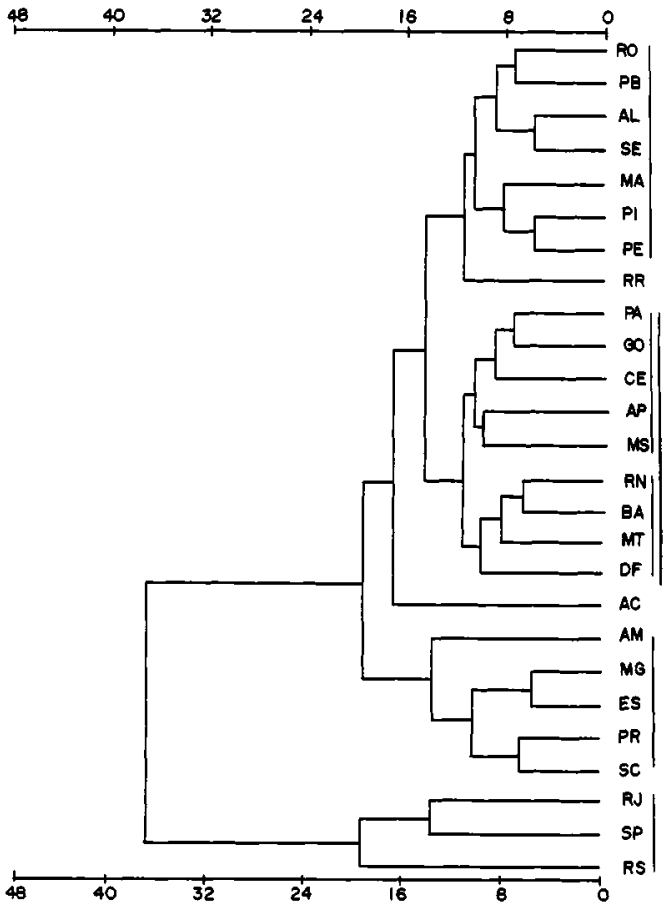

Figura 1. Dendrograma representando a Análise de Agrupamento dos Estados ou territórios para o conjunto das 13 variáveis (localizaçóes tumorais), para o ano de 1980, utilizando-se o algoritmo SL (Single Linkage).

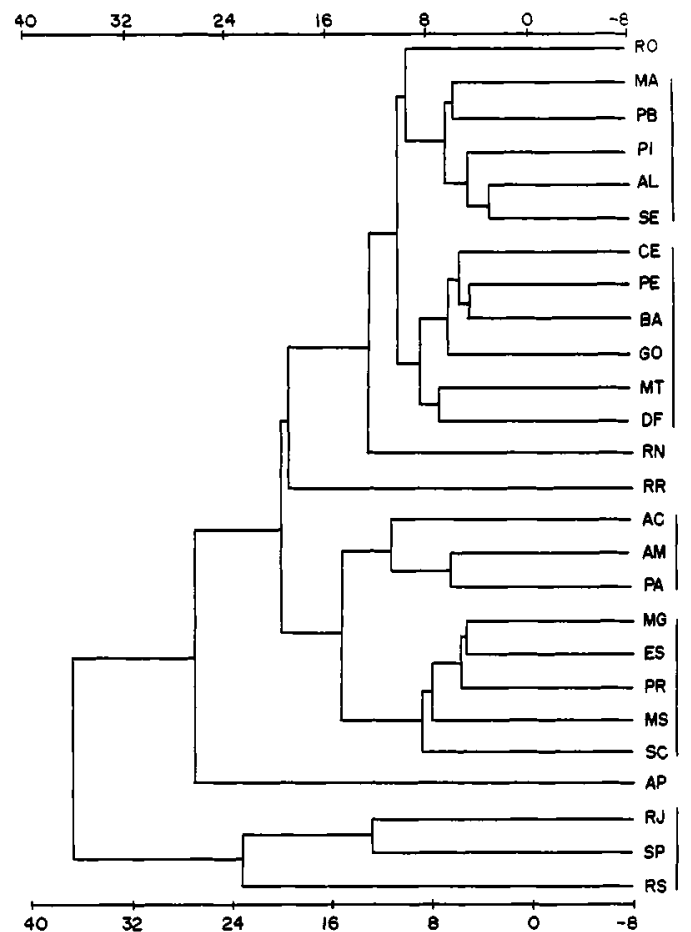

Figura 2. Dendrograma representando a Análise de Agrupamento dos Estados ou territórios para o conjunto das 13 variáveis (localizaçōes tumorais), para 0 ano de 1983, utilizando-se o algorítmo SL (Single Linkage).

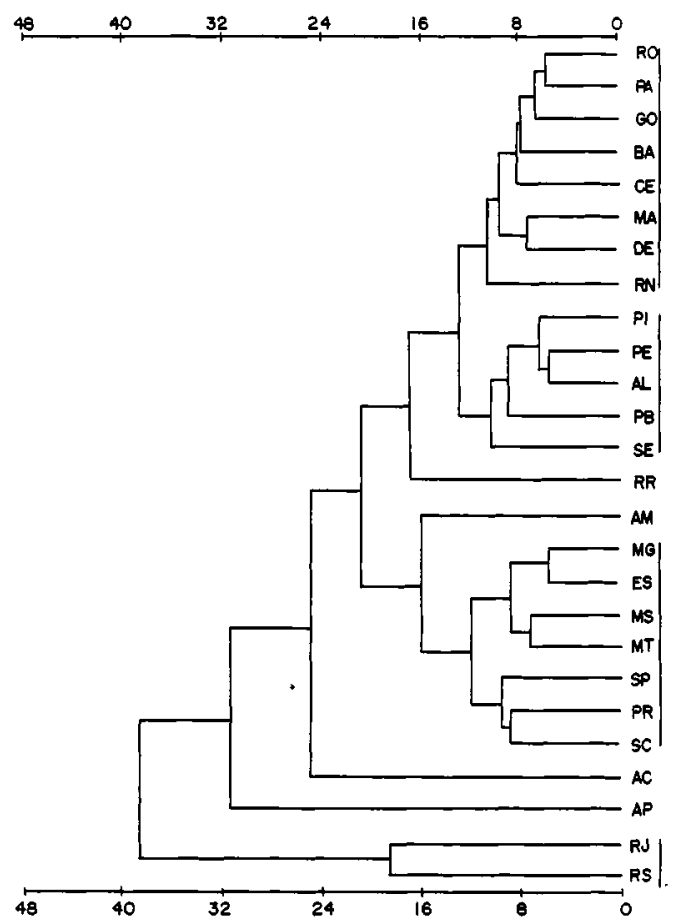

Figura 3. Dendrograma representando a Analise de Agrupamento dos Estados ou territórios para o conjunto das 13 variáveis (localizaçōes tumorais), para o ano de 1985, utilizando-se o algorítmo SL (Single Linkage) 


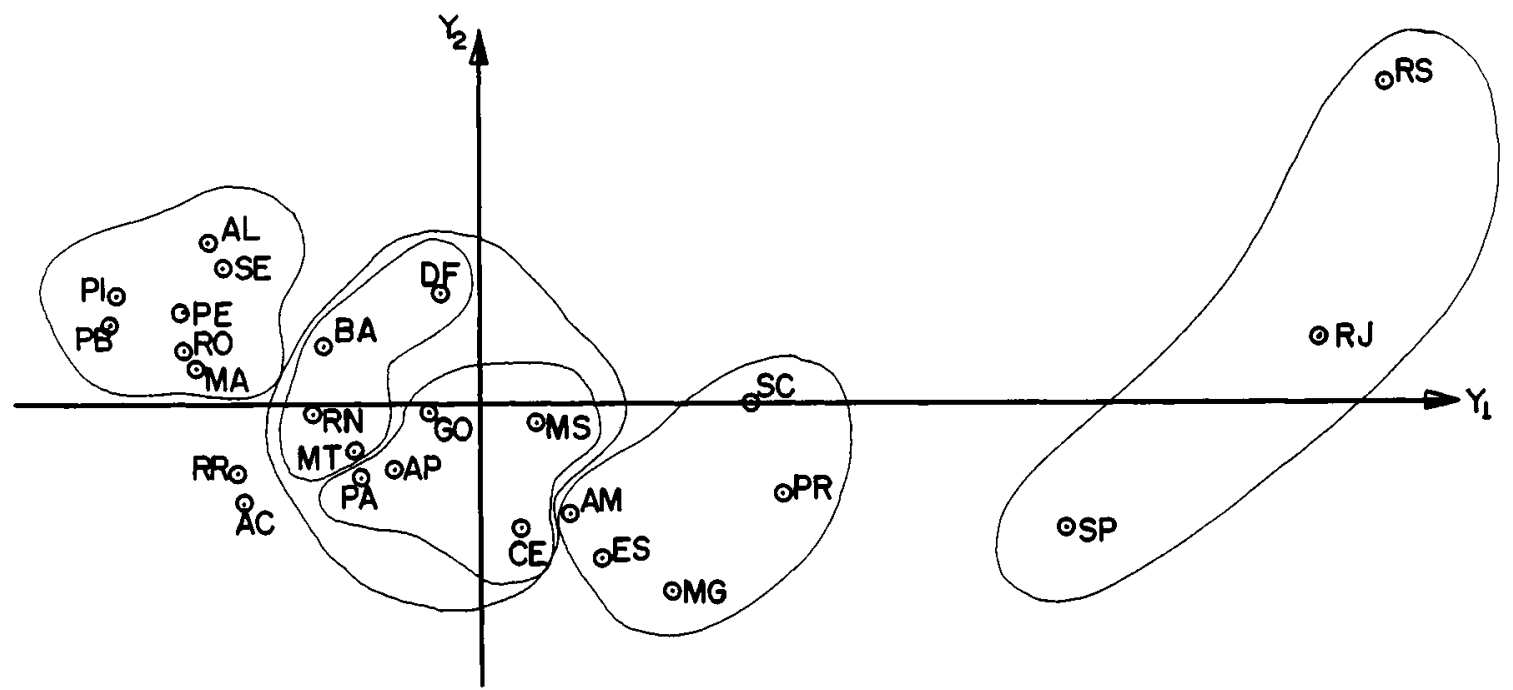

Figura 4. Ordenação das Unidades da Federaçāo (Estados e/ou territórios) pelos componentes principais, $Y_{1}$ e $Y 2$, para o ano de 1980.

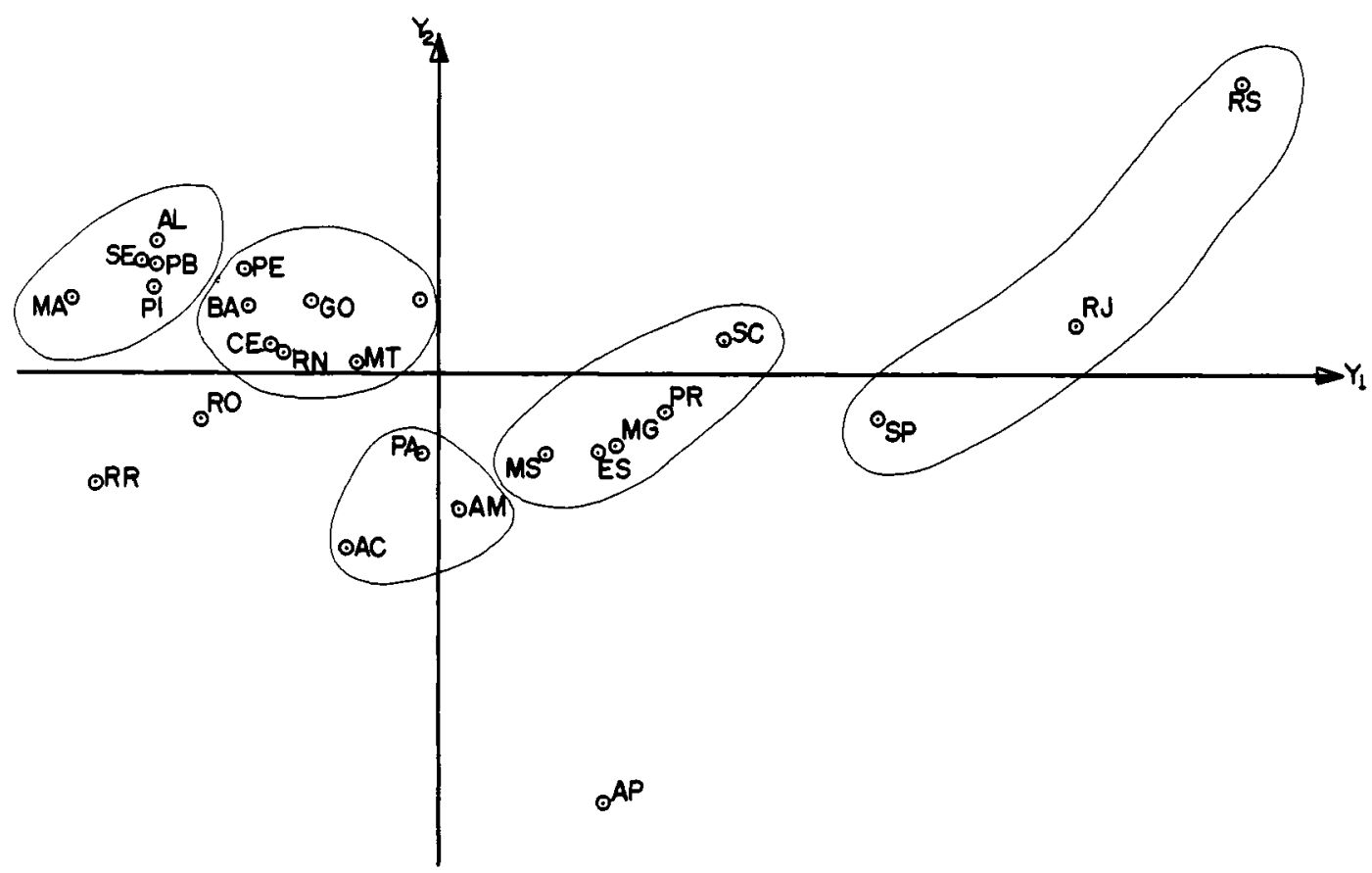

Figura 5. Ordenação das Unidades da Federação (Estados e/ou territórios) pelos componentes principais, Y1 e Y2, para o ano de 1983.

dante entre taxas de ocorrência de neoplasias malignas do pâncreas e do colon tem sido descrita também em outros países (Wynder e col. ${ }^{7}, 1967$ ).

$A$ análise de componentes principais mostrou que houve forte concordância quanto ao poder discriminatório das variáveis, entre os anos de 1980 e 1985, com algumas alteraçôs em 1983. Neoplasias malignas da traquêia/brônquios/pulmão foram indicadas nos três anos, como a variável com maior poder discriminatório. Seguiram-se as neoplasias malignas do estômago, do esôfago, do colon e do pâncreas. Os elementos com menor peso discriminatório foram as neoplasias malignas do colo uterino e as benignas/carcinoma "in situ"/outras. 


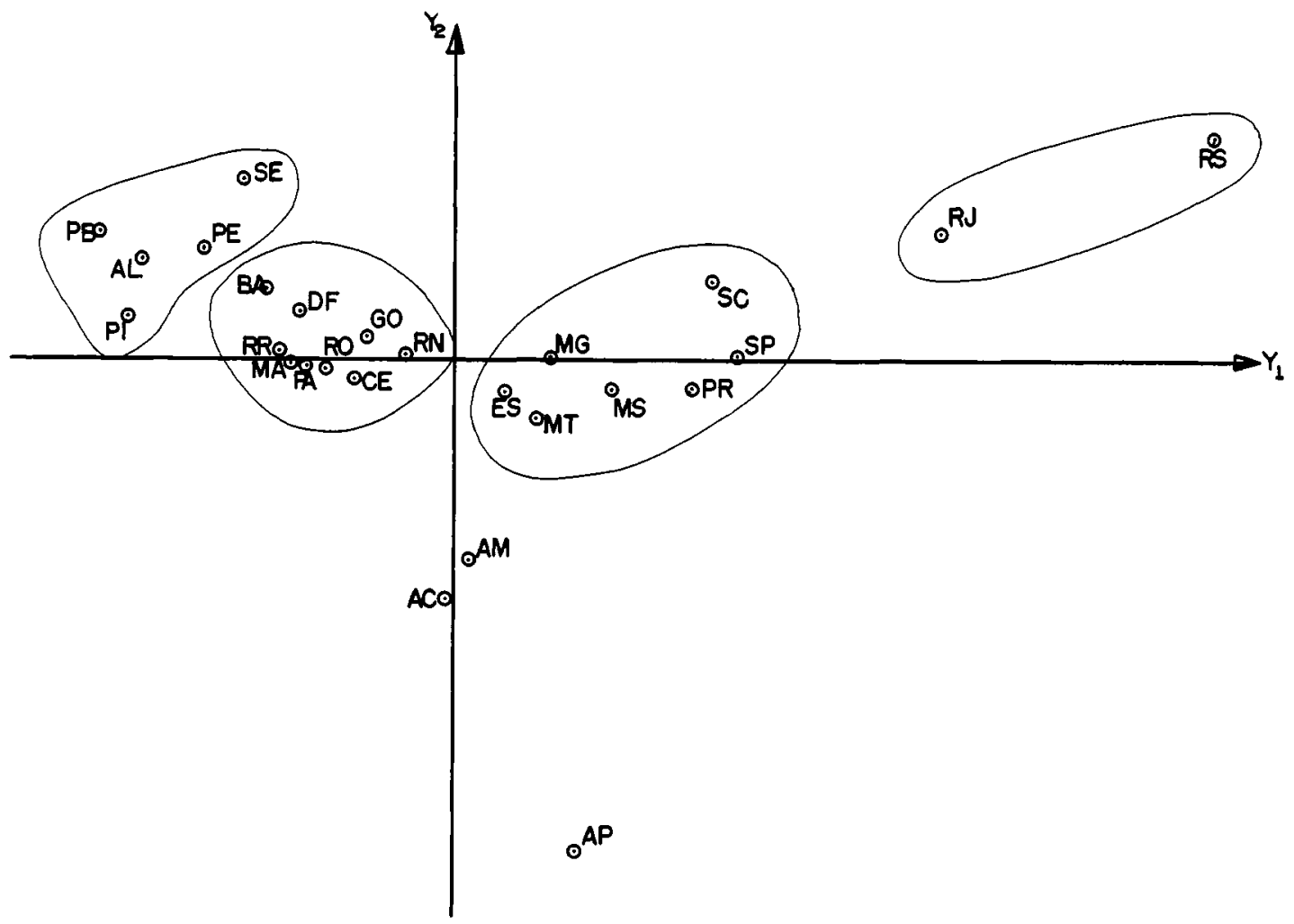

Figura 6. Ordenação das Unidades da Federação (Estados e/ou territórios) pelos componentes principais, Y1 e Y2, para o ano de 1985 .

O cálculo dos índices de mortalidade por neoplasias em todas as localizações, no conjunto dos Estados, permitiu detectar tendência de crescimento no número de mortes por neoplasias no Brasil, com o ano de 1985 (396,16 mortes/milhão de habitantes) apresentando um aumento de $3,49 \%$ em relação a 1980 (382,78 mortes/milhão de habitantes). Essa tendência de crescimento tem ocorrido também na maioria dos países (WHO ${ }^{16}, 1988$ ).

As neoplasias malignas do estômago revelaramse a principal causa de morte por neoplasias no Brasil, com média de 81,47 mortes/milhão de habitantes $(21,30 \%$ do total médio) e decréscimo de $5,31 \%$ no período estudado.Este resultado concorda com a tendência mundial de redução da taxas de mortalidade por neoplasia do estômago nas últimas décadas (WHO $\left.{ }^{16}, 1988\right)$.

Em seguida destacam-se as neoplasias malignas da traquéia/brônquio/pulmão, com média de 66,87 mortes/milhão de habitantes $(17,49 \%$ do total médio), com aumento de $15,22 \%$ no período. Vários autores relacionam este aumento mundial com a crescente urbanização que ocorre na maioria dos países, somada ao incremento no número de fumantes (Davis e Schwartz ${ }^{3}$, 1988; Montoro e Nogueira $\left.{ }^{9}, 1983\right)$. Os dados completos referentes
Tabela . Mortalidade relativa, participação no total médio e percentual de variação da mortalidade por neoplasias no Brasil, segundo a localização no período de 1980/1983/1985.

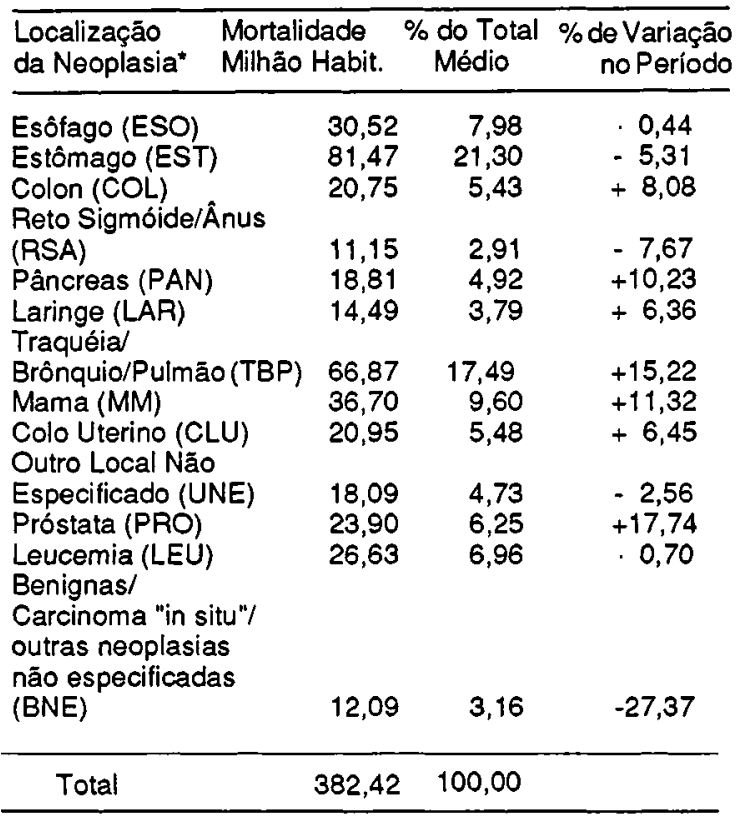


ao comportamento das variáveis no período estudado são apresentados na Tabela.

Examinando-se as diferentes regiões do país, percebe-se que os Estados das regiões Sul e Sudeste (em especial RS, RJ e SP) lideram as estatísticas de mortalidade no Brasil para a maioria das neoplasias. As exceções ficaram por conta das neoplasias malignas do estômago (AP na terceira posição), da laringe (AP e AM respectivamente na segunda e quinta posiçðes) e de colo uterino, onde AP, RR, AM, MA, e PI foram os Estados com maior índice de mortalidade para este tipo de neoplasia. Já entre as neoplasias malignas uterinas não especificadas, os Estados do AM e de MS são o terceiro e o quarto na estatística nacional.

Em relação às neoplasias benignas/carcinoma "in situ"/outras, os dados são bastante variáveis entre Estados e entre anos, sugerindo diferenças na precisão diagnóstica.

Destaque deve ser dado para os altos índices de mortalidade por neoplasias malignas do esôfago no Estado do RS, o qual, embora representando aproximadamente $7,3 \%$ da população brasileira, apresentou, em média , 18,3\% do total de mortes para esta patologia no período de 1980/1983/1985.

Vale ressaltar, ainda uma vez mais, que a maior ocorrência de mortalidade por neoplasias nas regiões Sul e Sudeste pode ser um fato real ou ser devida a maior eficiência na coleta de informações nessas regiōes.

PINTO, F.G. \& CURI, P.R. [Mortality from neoplasms in Brazil (1980/1983/1985): grouping by States, behaviors and tendencies] Rev. Saúde públ., S. Paulo, 25:276-81, 1991. Mortality caused by neoplasms in Brazil was examined by means of official Ministry of Health data covering 26 of the Federal Units and 13 different tumoral sites and referring to the years 1980,1983 and 1985. Both cluster analyses and those of principal components have shown heterogenous behaviour as between the different regions of the country in relation to the 13 variants studied. The main discriminatory elements are the trachea/bronchus/lung malign neoplasms followed by those of stomach, pancreas, colon and larynx. Complementary analyses have demonstrated a tendency to an increase in the mortality rate due to prostate malign neoplasms $(17.74 \%)$, followed by those of trachea/bronchus/lung (15.22\%), breast (11.32\%), pancreas $(10.23 \%)$, colon ( $8.08 \%)$, uterine colon (6.45\%) and larynx (6.36). There has been a decrease of the mortality due to benign neoplasms/carcinoma "in situ"/others (27.37\%), malign rectus neoplasms of the sigmoide/anus $(7.67 \%)$, stomach $(5.31 \%)$, of other non-specific locations in the uterus $(2.56 \%)$, of leukaemia $(0.70 \%)$ and malign neoplasms of the oesophagus $(0.44 \%)$. Maling neoplasms of the stomach have been the main cause of cancer mortality in Brazil accounting for $21.27 \%$ of the mean total, followed by of the malign neoplasms trachea/bronchus/lung
( $17.52 \%$ of the general average). The mortality rates by esophageal malign neoplasms in Rio Grande do Sul is stressed.

Keywords: Neoplasms, mortality. Residence characteristics.

\section{Referências Bibliográficas}

1.BRUMINI, R. Câncer no Brasil: dados histopatologicos: 1976-80. Rio de Janeiro, Campanha Nacional de Combate ao Câncer. Ministério da Saúde, 1982.

2.CAMPBELL, H. Cancer mortality in Europe: site-specific patterns and trends; 1955 to 1974. Wld Hith Statist. Quart., 33: 241-80, 1980.

3.DAVIS, D.L. \& SCHWARTZ, J. Trends in cancer mortality: US white males and females: 1968-83. Lancet, 1: 633$5,1988$.

4.ESTEVES, R.A.; GOES, J.S.; ALVAREZ, C.A. Oncologia: manual para el control del cancer en América Latina. Buenos Aires, Edit. Médica Panamericana, 1978.

5.LILIENFELD, A.M.; PEDERSEN, E.; DOWD, J. E. Cancer epidemiology: methods of study. Baltimore, Md, Johns Hopkins, 1967.

6.MINISTÉRTO DA SAÚDE. Secretaria Nacional de Ações Básicas de Saúde. Divisão Nacional de Epidemiologia. Estatisticas de mortalidade: Brasil - 1980. Brasília, Centro de Documentação do Ministério da Saúde, 1984.

7.MINISTÉRIO DA SAÚDE. Secretaria Nacional de Ações Básicas de Saúde. Divisão Nacional de Epidemiologia. Estatísticas da mortalidade: Brasil - 1983. Brasília, Centro de Documentaçāo do Ministério da Saúde, 1987.

8.MINISTÉRIO DA SAÚDE. Secretaria Nacional de Açōes Básicas de Saúde. Divisão Nacional de Epidemiologia. Estatisticas de mortalidade : Brasil - 1985. Brasilia, Centro de Documentação do Ministério da Saúde, 1989.

9.MONTORO, A.F \& NOGUEIRA, D.P. Meio ambiente e câncer. São Paulo, T. A. Queiroz, 1983.

10.PARKIN, D.M.; STJERNSWARD, J.; MUR, C.S. Estimates of the world wide frequency of twelve major cancers. Bull. Wld Hlth Org., 62: 163-82, 1984.

11.PERCY, C.; STAWEK, E.; GLOECKLER, L. Accuracy of cancer death certificates and its effect on cancer mortality statistics. Amer.J.publ. Hlth, 71: 242-50, 1981.

12.POSAS, $C$. Epidemiologia e sociedade: heterogeneidade estrutural e saúde no Brasil. São Paulo, Edit. Hucitec, 1989.

13.SILVA, M.G.C. da Câncer em Fortaleza: 1978-1980. Fortaleza, Instituto e Registro de Câncer do Ceará, 1982.

14.SNEATH, P.H.A. \& SOKAL, R.R Numerical taxonomy. San Francisco, W.H. Freeman, 1973.

15.SOLIS, C.V.; CERVANTES,M.L.; TONEY,S.V. Principales características epidemiologicas de la mortalidad por cancer en México. Saludpubl.México, 28:543-50, 1986.

16.WORLD HEALTH STATISTIC ANNUAL. Geneva, World Health Organization, 1988.

17.WYNDER, E.L.; HYAMS, A.; SHIGEMATSU, T. Correlations of international cancer death rates: an epidemiological exercise. Cancer, 20: 113-26, 1967.

Recebido para publicaçăo em 17/10/1990 Reapresentado em 15/04/1991 Aprovado para publicaçāo em 16/04/1991. 